\title{
Why mapping the brain matters
}

\author{
Obtaining anatomical maps and molecular information of brain circuits and their activity \\ patterns in relation to specific behaviors is instrumental to understanding brain function.
}

It is surprising how little we know about our brains and how they work, despite centuries of studying this fascinating organ. What exactly makes understanding the brain so difficult?

Through experimental investigation and observation, scientists have obtained a reasonable understanding of how most organs function. This knowledge has been instrumental to our understanding of the basis of diseases that affect these organs and for guiding treatments to cure them. Take the heart, for example. Using physiological, anatomical and molecular methods, it has been possible to make hypotheses of how its bloodconduction system operates, and to test and verify these hypotheses through experimentation. Why has this approach not worked in the brain?

Brains are incredibly complex in terms of the number and diversity of cells they contain. Moreover, neurons in the brain form hundreds of connections with other neurons, and these connectivity patterns can span very distal areas of the brain. It is the activity of connected cellular ensembles in the brain that is thought to drive our actions, store our memories, produce our thoughts and help us perceive the world. But the 'code' behind this form of information storage remains largely a mystery.

Progress has been made, particularly in the last few decades, and certain well-studied circuits are wellunderstood and have led to valid functional models. However, this mechanistic knowledge is sparse and has not yet led to the types of general principles about how the brain codes information that could lead to a more global understanding of this organ's function. Both theorists and experimental neuroscientists increasingly agree that we need more data to improve our hypotheses about how brains store information and retrieve it. This requires a better understanding of the components of the brain (neurons, glia and blood vessels at the least), how these components communicate with each other, and how their activities relate to specific behaviors and stimuli.

For many years, the technologies available to neuroscientists did not allow for such investigations, but this is rapidly changing. The Allen Brain Atlas and the Rockefeller University Gensat projects, among others, compile growing collections of gene expression and neuroanatomical data that can be of help in starting to decipher the molecular identities of cells in the brain.
Moreover, the methodology for imaging and tracing the connections of entire nervous systems at high resolution has now reached levels of automation and performance that will allow the mapping of portions of a mammalian brain or entire smaller brains at cellular resolution in a span of a few years, and techniques exist to add molecular information to those maps.

Relating this information to function and to specific behavioral states is a harder challenge, but progress, in this regard as well, is being made quickly. Using optical technologies, one can image the activity of almost all the neurons in a small, transparent brain such as that of a zebrafish larva near-simultaneously while the animal performs specific behaviors. The translation of these methods to mammalian brains, however, poses challenges, and new technology will be needed-requiring collaboration between neuroscientists, physicists, engineers and nanotechnologists. Although it might be hard to record activity from entire neural circuits in even a small mammalian brain in the next few decades, it is very likely that-informed by data from simpler organisms and through improved anatomical and molecular information-progress will be made in deriving general principles and hypotheses that can be directly tested in brains more similar to our own.

We are on the verge of embarking on a large initiative embraced by the US government with the aim of unveiling the mysteries of our minds, and as a result we will likely see a deluge of new data. But these data, once collected, will not magically produce the answers we seek. Instead, neuroscientists will be challenged to make sense of the information and to use it cleverly to elaborate better hypotheses and experimental approaches for direct testing. For this, they will need to work with mathematicians and statisticians to analyze and interpret the data and obtain input from theorists to help postulate testable hypotheses and models.

In this issue, we present a Focus on Mapping the Brain in which experts review the latest technologies for mapping the brain of different species at various scales and discuss critical considerations that will likely influence the success of this endeavor. It is also our hope that these pages will stimulate and inspire the development of new and better methods, theories and experiments to help answer this difficult and yet most fascinating of questions. 